\title{
COGNITIVE SEMIOTICS
}

\section{EDITORS IN CHIEF}

Peer F. Bundgaard, Aarhus

\section{ASSOCIATE EDITORS}

Merlin Donald, Kingston, ON

Bruno Galantucci, New York, NY

Todd Oakley, Cleveland, $\mathrm{OH}$

Göran Sonesson, Lund

\section{EDITORIAL BOARD}

Per Aage Brandt, Cleveland, $\mathrm{OH}$

Seana Coulson, San Diego, CA

Terrence W. Deacon, Berkeley, CA

Shaun Gallagher, Memphis, TN

Jean Petitot, Paris

Frederik Stjernfelt, Aarhus

Eve Sweetser, Berkeley

Leonard Talmy, Buffalo, NY

Evan Thompson, Toronto, ON

Mark Turner, Cleveland, $\mathrm{OH}$

Patrizia Violi, Bologna

Jordan Zlatev, Lund

\section{ADVISORY BOARD}

Liliana Albertazzi, Trento

Chiara Ambrosio, London

Juana Isabel Marin-Arrese, Madrid

Bernard Baars, San Diego, CA
Alexander Bergs, Osnabrück

Luis Emilio Bruni, Aalborg

Roberto Casati, Paris

Roberto Flores, Mexico City

Rick Grush, San Diego, CA

David Herman, Columbus, $\mathrm{OH}$

Barend van Heusden, Groningen

Mark Johnson, Eugene, OR

Kalevi Kull, Tartu

Jean Lassègue, Paris

Ronald Langacker, San Diego, CA

Michael Leyton, New Brunswick, NJ

Dominic McIver Lopes, Vancouver, BC

Raymond A. Mar, Toronto, ON

Irene Mittelberg, Aachen

Frederic Nef, Paris

Douglas Niño, Bogotá

Martina Plümacher, Berlin

Roberto Poli, Trento

Andreas Roepstorff, Aarhus

Chris Sinha, Portsmouth

Jesper Sørensen, Aarhus

Mónica Tamariz, Edinburgh

Reuven Tsur, Tel Aviv

Kristian Tylén, Aarhus

Mikkel Wallentin, Aarhus

Wolfgang Wildgen, Bremen

\section{DE GRUYTER MOUTON}


ABSTRACTED/INDEXED IN Baidu Scholar · BDSL Bibliographie der deutschen Sprach- und Literaturwissenschaft · BLL Bibliographie Linguistischer Literatur · Cabells Journalytics · CNKI Scholar (China National Knowledge Infrastructure) · CNPIEC: cnpLINKer Dimensions EBSCO (relevant databases) · EBSCO Discovery Service · Genamics JournalSeek · Google Scholar · IBR (International Bibliography of Reviews of Scholarly Literature in the Humanities and Social Sciences) · IBZ (International Bibliography of Periodical Literature in the Humanities and Social Sciences) $\cdot \mathrm{J}$-Gate JournalTOCs · KESLI-NDSL (Korean National Discovery for Science Leaders) · Linguistic Bibliography · Microsoft Academic · MLA International Bibliography · MyScienceWork · Naver Academic $\cdot$ Naviga (Softweco) · Primo Central (ExLibris) · ProQuest (relevant databases) · PSYNDEX · Publons · QOAM (Quality Open Access Market) · ReadCube · SCImago (SJR) - SCOPUS · Semantic Scholar $\cdot$ Sherpa/RoMEO $\cdot$ Summon (ProQuest) $\cdot$ TDNet $\cdot$ Ulrich's Periodicals Directory/ulrichsweb - WanFang Data $\cdot$ WorldCat (OCLC) · Yewno Discover

e-ISSN 2235-2066

All information regarding notes for contributors, subscriptions, Open access, back volumes and orders is available online at www.degruyter.com/cogsem

RESPONSIBLE EDITOR Peer F. Bundgaard, Aarhus University, Center for Semiotics Jens Chr. Skous Vej 7, 8000 Aarhus, Denmark, e-mail: sempb@dac.au.dk

PUBLISHER Walter de Gruyter GmbH, Berlin/Boston, Genthiner Straße 13, 10785 Berlin, Germany JOURNAL COORDINATOR Esther Markus, De Gruyter, Genthiner Straße 13, 10785 Berlin, Germany, Tel.:+49 (0)30 26005 127, e-mail: esther.markus@degruyter.com

RESPONSIBLE FOR ADVERTISEMENTS Markus Kügel, De Gruyter, Rosenheimer Str. 143, 81671 München, Germany. Tel.: +49 8976 902-424, e-mail: anzeigen@degruyter.com

(C) 2021 Walter de Gruyter GmbH, Berlin/Boston

TYPESETTING TNQ Technologies, Chennai, India 